\title{
DOCKING ANTIOXIDANT ACTIVITY ON HYDROXY (DIPHENYL) ACETICACID AND ITS DERIVATIVES
}

\author{
SUDHA R ${ }^{1 *}$, NITHYA G ${ }^{1}$, BRINDHA DEVI P² ${ }^{2}$ CHARLES C KANAKAM ${ }^{3}$ \\ ${ }^{1}$ Department of Chemistry, School of Basic Sciences, Vels University, Chennai, Tamil Nadu, India. ${ }^{2}$ Department of Bio-engineering, \\ School of Engineering, Vels University, Chennai, Tamil Nadu, India, ${ }^{3}$ Depatment of Chemistry, Formerly Presidency College, \\ University of Madras, Tamil Nadu, India. Email: rajendran.sudha7@gmail.com
}

Received: 06 March 2017, Revised and Accepted: 13 April 2017

ABSTRACT

Objectives: The antioxidant activity of the synthesized compounds along with the standard compound for comparison is reported. There is comparison of binding analysis and the ligand interaction of the compound.

Methods: The protein crystal structure complexed with 4-methyl-6-[2-(5-morpholin-4-ylpyridin-3-yl)ethyl]pyridin-2-amine inhibitor was selected from Protein Data Bank (5FVP) for our study.

Results: The docking studies and structure-activity relationship reveals that the compound 2'-chloro-4-methoxy-3nitro benzilic acid after three different docking strategies reveals that the score was found to be higher compared with others.

Conclusion: Based on the in vitro antioxidant results, the compounds synthesized were investigated for the molecular docking study to identify the amino acid interactions in the active site pocket of nitric oxide synthase enzyme. Based on the docking score results, all the compounds were oriented toward the active site pocket occupied by the cocrystallized ligand.

Keywords: Docking, Antioxidant activity, Stacking interaction.

(C) 2017 The Authors. Published by Innovare Academic Sciences Pvt Ltd. This is an open access article under the CC BY license (http://creativecommons. org/licenses/by/4. 0/) DOI: http://dx.doi.org/10.22159/ajpcr.2017.v10i7.18299

\section{INTRODUCTION}

Docking is frequently used to predict the binding orientation of small molecule drug candidates to their protein targets to predict the affinity and activity of the small molecule [1-5]. Hence, docking plays an important role in the 158 rational drug designs. Given the biological and pharmaceutical significance of molecular docking, considerable efforts have been directed toward improving the methods used to predict docking. A binding interaction between a small molecule ligand and enzyme protein may result in activation or inhibition of the enzyme. If the protein is a receptor, ligand binding may result in agonism or antagonism. Docking is molecular virtual screening, a computational method where the compounds could be assessed for their potential to bind specific sites on target molecules such as proteins, was employed in the study [6]. The pharmacokinetic properties were also predicted for future perspective of the small molecule compounds. Molecular docking studies are used to determine the interaction of two molecules and to find the best orientation of ligand which would form a complex with overall minimum energy. The small molecule, known as ligand usually fits within protein's cavity which is predicted by the search algorithm. These protein cavities become active when they come in contact with any external compounds and are thus called as active sites commonly used in the field of drug design-most drugs are small organic molecules. In living organism, the reactive oxygen species are formed as by-product of metabolic reactions, which cause diseases due to the initiation of oxidative reaction in biomolecules [7-9]. The reactive oxidation species formed during the metabolic process are nullified by the antioxidant substance having high antiradical power $[10,11]$.

Protein preparation and active site cavity validation of the protein nitric oxide synthase (NOS)

The retrieved protein was prepared and hydrogen atoms, water molecules were removed and prepared. The crystal ligand tetracyclic aza-amide was found to show hydrogen bonding interactions with
Glu592 and Trp587 amino acid residues. To validate the docking program, the crystal ligand was prepared and redocked on to the grid of the active site. The docking score was found to be $-6.670 \mathrm{kcal} / \mathrm{mol}$. The diagrammatic representation of reference ligand is shown in Fig. 1. The active site of the protein was located, and grid files were generated using receptor grid generation panel. Grid size was found to be $20 \AA$ and is used for all docking analysis.

The reference docking results showed that the compound exhibited similar interactions as that of the original crystal structure which was further confirmed with redocking with the RMSD of $1.85 \AA$. The superimposition of docked pose and original crystal ligand pose of tetracyclic aza-amide is presented in Fig. 2.

\section{Docking analysis}

The selected ligands were docked into the tetracyclic aza-amide active site using the default grid and finally selected as hits from high

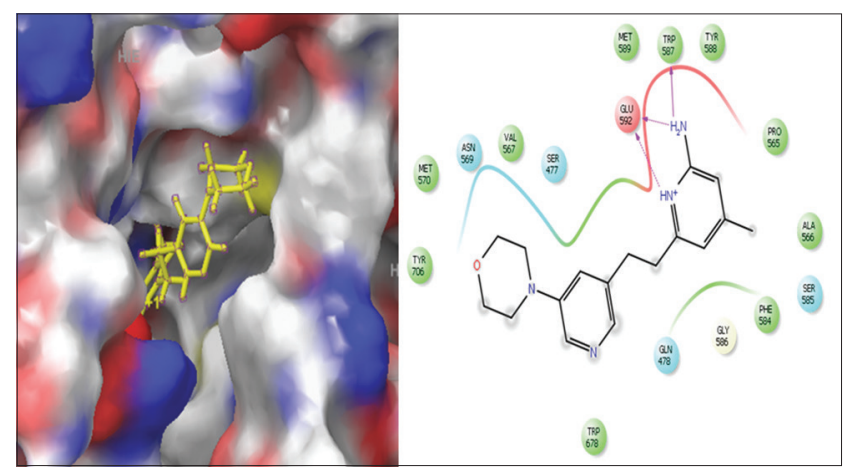

Fig. 1: Binding analysis and the ligand interaction of the reference ligand 
throughput virtual screening with the criteria of docking score were further docked using Glide standard precision docking module, and filtered ligands were subjected to Glide XP docking analysis. The resulted compounds with docking scores were found to be in the range of -8.061 to $-4.272 \mathrm{kcal} / \mathrm{mol}$. Although the docking score did not indicate the compound's binding affinity, it was helpful in prioritizing/ranking the ligands. Final shortlisting of hit compounds was based on visual inspection of the important amino acid residues involved in binding that included hydrogen bonding with Asn569. The docking score for the shortlisted compounds is tabulated in Table 1.

\section{RESULTS AND DISCUSSIONS}

There were many crystal structures published in the Protein Data Bank (PDB) (www.rcsb.org/pdb) on NOS in complexes with ligands and fragments developed by fragment-based design with resolutions ranging from 2.1 to $2.14 \AA$. As we attempted to utilize the crystal structure of NOS in complex with an inhibitor nitric oxide (PDB ID: 5FVP) with $2.1 \AA$ resolution for further active site validation and docking analysis.

NOS enzyme crystal structure complexed with inhibitor was taken for our study to discover novel hit molecule for antioxidant drug discovery. The reference ligand was docked into the active site of the enzyme, and the docking score was found to be $-6.637 \mathrm{kcal} / \mathrm{mol}$. The amino group of reference ligand was found to interact with positively charged amino acid Glu592 and non-polar amino acid Trp587.

Later, the synthetic compounds selected for our study was made to dock into the active site pocket of the enzyme NOS and found that the compound 2'chloro-4-methoxy-3-nitro-benzilic acid was found to be $-8.061 \mathrm{kcal} / \mathrm{mol}$. The closer analysis of the compound was analyzed and found that the compound was found to interact with the amino acid Ser457 and the benzyl group is stacked with the non-polar amino acid Trp409. The 3-dimensional view of this molecule reveals that the compound was well fitted into the active site cavity which made this molecule more effective binding than the reference ligand. Furthermore, the nitro group and methoxyphenyl group was well surrounded by the non-polar amino acids. This results also signifies the in vitro antioxidant activity. The binding analysis and the ligand interaction diagram was depicted in the Fig. 3

The compound 2,2'-dichlorobenzilic acid showed the docking score of $-7.374 \mathrm{kcal} / \mathrm{mol}$ to further discuss about this compound binding analysis and interactions, the amino acid Ser457 donates one hydrogen atom to the compound and the chloro benzilic group was found to be interact with two stacking interaction with non-polar amino acids Trp409 and Phe584. Furthermore, the compound is fully surrounded by the non-polar amino acids such as Val567, Ala566, and Ile459 which made this compound possess better docking score than others. The binding analysis and the docking score of the compound were depicted in Fig. 4.

The compound 4,4' dibromobenzilic acid showed the docking score of $-7.083 \mathrm{kcal} / \mathrm{mol}$. Further, the structure-activity relationship of this compound reveals that the bromophenyl group is showing a stacking interaction with Trp409. Due to the presence of bulky halogenic group present on the both the side of this compound, the compound tends to lose its activity on binding with the enzyme [12]. The binding analysis and the ligand interaction of the compound were depicted in Fig. 5.
The compound methyl benzilate possesses the docking score of $-6.290 \mathrm{kcal} / \mathrm{mol}$. The closer analysis of this compound shows that the two benzylic group was found to interact with non-polar amino acids Phe584 and Trp409. Furthermore, the carbonyl group of this compound found to interact with Ser457. The 3-dimensional representation of this molecule reveals that the compound is slightly away from the active site

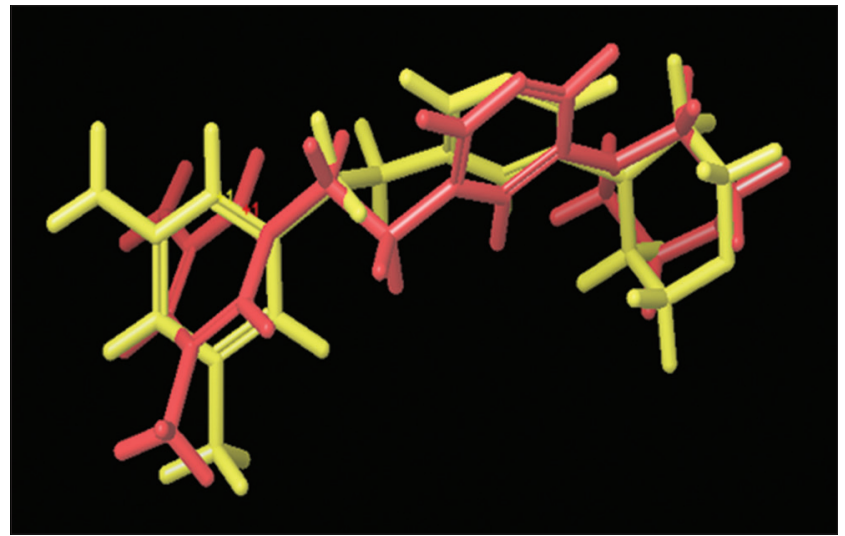

Fig. 2: Superimposition of docked pose of etracyclic aza-amide and original pose of tetracyclic aza-amide, where yellow color indicates the original ligand and the red color represents docked ligand
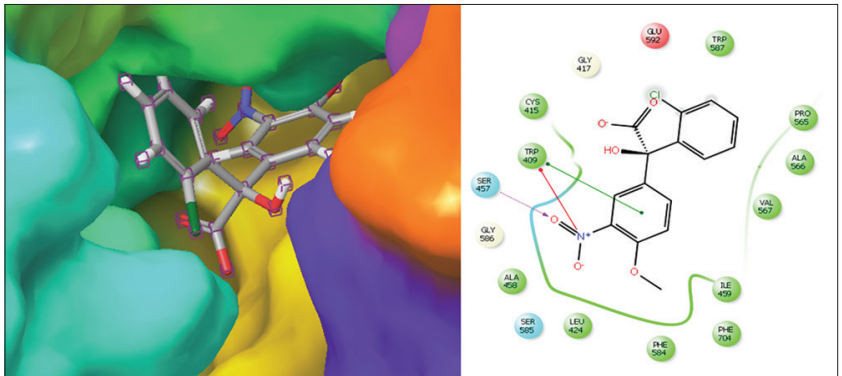

Fig. 3: The binding analysis and the ligand interaction for the compound 2'chloro-4-methoxy benzylic acid
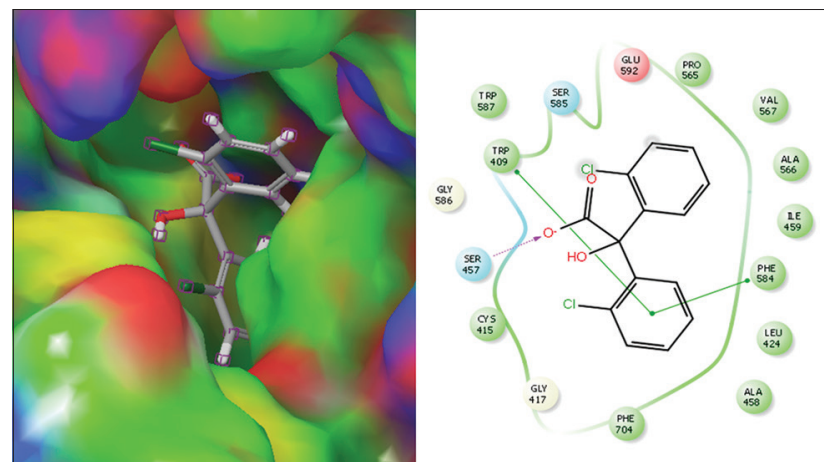

Fig. 4: The binding analysis and the ligand interaction for the compound 2,2' dichlorobenzilic acid

Table 1: Docking score and ligand interaction results for the synthesized compounds

\begin{tabular}{llll}
\hline S. No. & Compound name & Docking score kcal/mol & Ligand interaction \\
\hline 1. & 2'-chloro4-methoxy-3-nitro benzilic acid & -8.061 & Ser457, Trp409, Phe584 \\
2. & 2,2'-dichlorobenzilic acid & -7.374 & Ser457, Trp409, Phe584 \\
3. & $4,4^{\prime}$-dibromo benzilic acid & -7.083 & Trp409 \\
4. & Methyl benzilate & -6.290 & Trp409, Ser457 \\
5. & Diisopropyl ammounium benzilate & -6.035 & Trp409 \\
6. & Benzilic acid & -4.272 & Trp587 \\
\hline
\end{tabular}



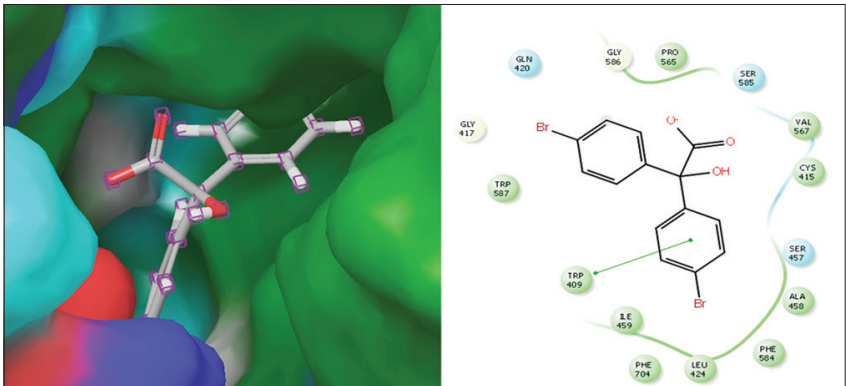

Fig. 5: The binding analysis and the ligand interaction of the compound 4,4'dibromobenzilic acid
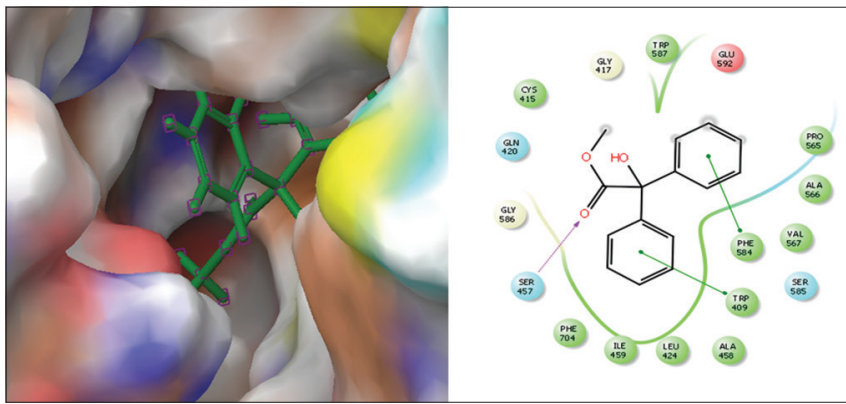

Fig. 6: The binding analysis and the ligand interaction of the compound methyl benzilate

which made this compound lesser active than others [13]. The binding analysis and the ligand interaction of the compound were depicted in Fig. 6.

The compound diisopropyl ammonium benzilate shows the docking score of $-6.035 \mathrm{kcal} / \mathrm{mol}$. The ligand interactions of this compound reveal that the benzyl group was found to interact with Trp409. Moreover, the compound was very far from the validated active site pocket which made this compound lesser active than the other compounds. The compound does not possess any other non-polar interactions other than Trp409 and Leu424. The binding analysis and the ligand interaction of this compound were depicted in Fig. 7.

The last compound benzylic acid was made to dock into the active site of the enzyme, and the docking score was found to be $-4.272 \mathrm{kcal} / \mathrm{mol}$. The hydroxyl group present in the compound was found to interact with the amino acid Trp587. Furthermore, the compound does not possess any other interaction apart from tryptophan. The benzyl group's present in this compound was well surrounded by non-polar interactions Val649 and Met589. Since this compound possesses only one interaction, the docking score was lesser than the other compounds. The binding analysis and docking score of this compound were depicted in Fig. 8.

\section{REFERENCES}

1. Bajorath J. Integration of virtual and high-throughput screening. Nat Rev Drug Discov 2002;1(11):882-94.

2. Walters WP, Stahl MT, Murcko MA. Virtual screening-an overview.

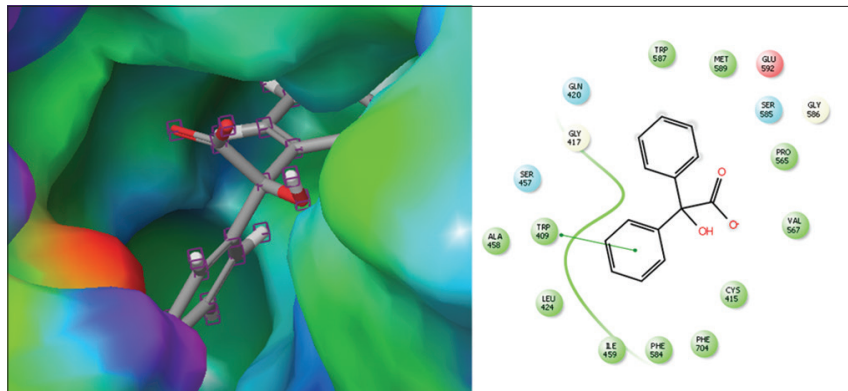

Fig. 7: The binding analysis and the ligand interaction for the compound diisopropyl ammonium benzilate

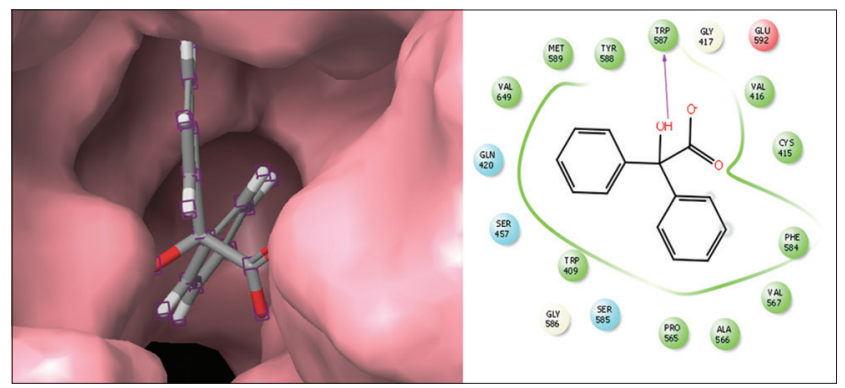

Fig. 8: The binding analysis and the ligand interaction for the compound benzylic acid

Drug Discov Today 1998;3(4):160-78.

3. Langer T, Hoffmann RD. Virtual screening: An effective tool for lead structure discovery? Curr Pharm Des 2001;7(7):509-27.

4. Gohlke H, Klebe G. Approaches to the description and prediction of the binding affinity of small-molecule ligands to macromolecular receptors. Angew Chem Int Ed Engl 2002;41(15):2644-76.

5. Kuntz ID, Blaney JM, Oatley SJ, Langridge R, Ferrin TE. A geometric approach to macromolecule-ligand interactions. J Mol Biol 1982;161(2):269-88

6. Hajduk PJ, Greer J. A decade of fragment-based drug design: Strategic advances and lessons learned. Nat Rev Drug Discov 2007;6(3):211-9.

7. Bhattacharya $\mathrm{S}$. Reactive oxygen species and cellular defense system. DOI: 10.1007/978-81-322-2035-0_2.

8. Briegar K, Schiavone S, Miller FJ, Krause KH. Reactive oxygen species: From health to disease. Swiss Med Wkly 2012. DOI: 10.4414/ smw.2012.13659.

9. Weidinger A, Kozlov AV. Biological activities of reactive oxygen and nitrogen species: Oxidative stress versus signal transduction. Biomolecules 2015;5(2):472-84

10. Rahman T, Hosen I, Islam MM, Shekar HU. Oxidative stress and human health. Adv Biosci Biotechnol 2012;3:997-1019.

11. Chang HY, Ho YL, Sheu MJ, Li YH. Antioxidants and free radical scavenging activity of Phellinus merrillii extracts. Bot Stud 2007;48:407-17.

12. Bairam R, Muppavarapu SM, Sreekanth S. Synthesis, characterization, biological evaluation and docking of some novel substituted 1, 3-thiazine derivatives. Int J Pharm Pharm Sci 2017;9(3):233-42.

13. Geetha P, Kumar BL, Indra U, Sheetal BP. Int J Pharm Pharm Sci. DOI: 10.22159/ijpps.2017v9i3.16485. 